\title{
INNER AMENABILITY AND FULLNESS
}

\author{
MARIE CHODA
}

\begin{abstract}
Let $G$ be a countable group which is not inner amenable. Then the $\mathrm{II}_{1}$-factor $M$ is full in the following cases:

(1) $M$ is given by the group measure space construction from a triple $(X, \mu, G)$ with respect to a strongly ergodic measure preserving action of $G$ on a probability space $(X, \mu)$.

(2) $M$ is the crossed product of a full $\mathrm{II}_{1}$-factor by $G$ with respect to an action.
\end{abstract}

1. Introduction. The set of $\mathrm{II}_{1}$-factors is decomposed into two classes. The first is the set of $\mathrm{II}_{1}$-factors which have property $\Gamma$ due to Murray and von Neumann, and which contains the set of hyperfinite $\mathrm{II}_{1}$-factors. The second is the set of $\mathrm{II}_{1}$-factors which are called full [4]. Most known examples of $\mathrm{II}_{1}$-factors are given as one of the following algebras (or composition of those): (1) the group von Neumann algebra associated with an ICC (i.e., infinite conjugacy class) group, (2) the group measure space construction algebra, or more generally, (3) the crossed product of a given von Neumann algebra by an automorphism group.

In [6], Effros introduced the notion "inner amenability" for countable groups. He showed that if a countable ICC group $G$ is not inner amenable then the group factor (associated with $G$ ) is full.

In this paper, we shall show that similar results hold for algebras of the above types (2) and (3), so that "noninner amenability" for groups is a desirable property in order to construct a full $\mathrm{II}_{1}$-factor.

2. Full $\mathrm{II}_{1}$-factor. Let $N$ be a $\mathrm{II}_{1}$-factor with the canonical trace $\tau$. Then the following three statements are equivalent [4]: (4) $N$ is full, (5) $N$ has not property $\Gamma$, and (6) a (operator norm) bounded sequence $\left(x_{n}\right)$ in $N$, for which $\left\|x_{n} y-y x_{n}\right\|_{2} \rightarrow 0$ for all $y \in N$, satisfies $\left\|x_{n}-\tau\left(x_{n}\right) 1\right\|_{2} \rightarrow 0$, where $\|x\|_{2}=\tau\left(x^{*} x\right)^{1 / 2}$ for an $x \in N$.

A countable group $G$ is inner amenable if and only if there is a sequence $\left(\xi_{n}\right)$ in $l^{2}(G)$ (the Hilbert space of square summable functions on $G$ ) such that $\left\|\xi_{n}\right\|_{2}=1$, $\xi_{n}(1)=0$ and $\Sigma_{h \in G}\left|\xi_{n}\left(g h g^{-1}\right)-\xi_{n}(h)\right|^{2} \rightarrow 0$ for all $g \in G$, where 1 is the identity of $G$ [6]. The following groups are not inner amenable: (7) the free group with two generators by [6], (8) the free product of two nontrivial groups not both of order 2, and (9) an ICC group with Kazhdan's property T ([8]) by [1].

\footnotetext{
Received by the editors November 17, 1981 and, in revised form, April 20, 1982. 1980 Mathematics Subject Classification. Primary 46L35; Secondary 22D25, 46L10.

Key words and phrases. Factor, group algebra, crossed product, ergodicity.

${ }^{1}$ Partially supported by KAKENHI.
} 
Let $N$ be a finite von Neumann algebra acting on a separable Hilbert space $H, \tau$ a faithful normal trace on $N$ such that $\tau(1)=1$, and $G$ a countable group of $\tau$-preserving automorphisms on $N$. The action of $G$ is said to be ergodic on $N$ if $N^{G}=\{x \in N ; g(x)=x$ for all $g \in G\}=\mathbf{C l}$, and strongly ergodic on $N$ if a bounded sequence $\left(x_{n}\right)$ in $N$ for which $\left\|g\left(x_{n}\right) \rightarrow x_{n}\right\|_{2} \rightarrow 0$ for all $g \in G$, satisfies necessarily $\left\|x_{n}-\tau\left(x_{n}\right) 1\right\|_{2} \rightarrow 0$ (cf. [5]). A strongly ergodic action is ergodic. If $G$ has property $\mathrm{T}$, then an ergodic action of $G$ is strongly ergodic [2]. Put

$$
\begin{array}{r}
(\pi(a) \xi)(g)=g^{-1}(a) \xi(g) \text { and } \quad(v(g) \xi)(h)=\xi\left(g^{-1} h\right) \\
\left(a \in N, g, h \in G, \xi \in l^{2}(G, H)\right),
\end{array}
$$

where $l^{2}(G, H)$ is the Hilbert space of square summable $H$-valued functions on $G$. Then $\pi$ (resp. $v$ ) is a representation of $N$ (resp. $G$ ) on $l^{2}(G, H)$ such that $v(g) \pi(a) v(g)^{*}=\pi(g(a))$ for all $g \in G$ and $a \in N$. The crossed product $M$ of $N$ by $G$ is the von Neumann algebra generated by $\pi(N)$ and $v(G)$. Let $e$ be the faithful normal expectation of $M$ onto $\pi(N)$ such that $e(v(g))=0$ for $g \neq 1$ (see [7], for example). Then $M$ is a finite von Neumann algebra with a faithful normal trace $\tau \cdot e$ and each $x \in M$ has a unique expansion $x=\Sigma_{g \in G} x(g) v(g)(x(g) \in \pi(N)$ for all $g \in G)$ in the sense of $\|\cdot\|_{2}$-metric convergence.

It is known that ergodicity is essential to the group measure space construction for a factor. The strong ergodicity is necessary for the group measure space construction of a full $\mathrm{II}_{1}$-factor [3].

THEOREM. Let $N$ be a finite von Neumann algebra with a faithful normal trace $\tau$ such that $\tau(1)=1, G$ a countable group of $\tau$-preserving automorphisms of $N$ and $M$ be the crossed product of $N$ by $G$. Assume that $G$ is not inner amenable. Then

(i) A sequence $\left(x_{n}\right)$ in $M$, for which $\left\|x_{n}\right\|_{2}=1$ for all $n$ and $\left\|x_{n} v(g)-v(g) x_{n}\right\|_{2}$ $\rightarrow 0$ for all $g \in G$, satisfies $\left\|x_{n}-e\left(x_{n}\right)\right\|_{2} \rightarrow 0$. In particular, $v(G)^{\prime} \cap M=\pi\left(N^{G}\right)$.

(ii) If the action of $G$ is strongly ergodic on $N$, then $M$ is a full $\mathrm{II}_{1}-$ factor.

Proof. (i) Let $\left(x_{n}\right)$ be a sequence in $M$ for which $\left\|x_{n}\right\|_{2}=1$ for all $n$ and $\left\|x_{n} v(g)-v(g) x_{n}\right\|_{2} \rightarrow 0$ for all $g \in G$. Let $x_{n}=\Sigma_{g \in G} x_{n}(g) v(g)\left(x_{n}(g) \in \pi(N)\right)$ be the Fourier expansion of $x_{n}$. For each $n$, put $\xi_{n}=\Sigma_{g \in G}\left\|x_{n}(g)\right\|_{2} \delta(g)$, where $\delta(g)$ is the characteristic function of $\{g\}$. Then $\left(\xi_{n}\right)$ is a sequence of unit vectors in $l^{2}(G)$ and satisfies

$$
\begin{aligned}
\sum_{h \in G}\left|\xi_{n}\left(g h g^{-1}\right)-\xi_{n}(h)\right|^{2} & =\sum_{h \in G}\left|\left\|x_{n}\left(g h g^{-1}\right)\right\|_{2}-\left\|x_{n}(h)\right\|_{2}\right|^{2} \\
& =\sum_{h \in G}\left|\left\|x_{n}\left(g h g^{-1}\right)\right\|_{2}-\left\|v(g) x_{n}(h) v(g)^{*}\right\|_{2}\right|^{2} \\
& \leqslant \sum_{h \in G}\left\|x_{n}\left(g h g^{-1}\right)-v(g) x_{n}(h) v(g)^{*}\right\|_{2}^{2} \\
& =\left\|x_{n} v(g)-v(g) x_{n}\right\|_{2}^{2} \rightarrow 0, \quad \text { for all } g \in G .
\end{aligned}
$$


If $\left\|\xi_{n}-\xi_{n}(1) \delta(1)\right\|_{2}$ does not converge to zero, there are an $\alpha>0$ and a subsequence $\left(\xi_{n(k)}\right)$ of $\left(\xi_{n}\right)$ such that $\left\|\xi_{n(k)}-\xi_{n(k)}(1) \delta(1)\right\|_{2} \geqslant \alpha$ for all $k$. For each $k$, put

$$
\zeta_{k}=\left(\xi_{n(k)}-\xi_{n(k)}(1) \delta(1)\right) /\left\|\xi_{n(k)}-\xi_{n(k)}(1) \delta(1)\right\|_{2} .
$$

Then the sequence $\left(\zeta_{k}\right)$ satisfies that $\left\|\zeta_{k}\right\|_{2}=1, \zeta_{k}(1)=0$ and

$$
\sum_{h \in G}\left|\zeta_{k}\left(g h g^{-1}\right)-\zeta_{k}(h)\right|^{2} \rightarrow 0 .
$$

This contradicts the noninner amenability of $G$. Hence $\left\|\xi_{n}-\xi_{n}(1) \delta(1)\right\|_{2} \rightarrow 0$. Therefore,

$$
\left\|x_{n}-e\left(x_{n}\right)\right\|_{2}^{2}=\sum_{g \neq 1}\left\|x_{n}(g)\right\|_{2}^{2}=\left\|\xi_{n}-\xi_{n}(1) \delta(1)\right\|_{2}^{2} \rightarrow 0 .
$$

(ii) If the action of $G$ is ergodic on $N$, then by (i) $M^{\prime} \cap M \subset v(G)^{\prime} \cap M=\pi\left(N^{G}\right)$ $=\mathbf{C} 1$. Hence $M$ is a finite factor. If $G$ is not ICC, there is an $h(\neq 1) \in G$ for which $\left\{g h g^{-1} ; g \in G\right\}$ is a finite set. Put $z=\Sigma_{g \in G} v\left(g h g^{-1}\right)$. Then $e(z)=0$ and $0 \neq z \in$ $v(G)^{\prime} \cap M=\mathbf{C l}$. This is a contradiction. Hence $G$ is an ICC group.

Therefore $M$ contains a $\mathrm{II}_{1}$-factor $v(G)^{\prime \prime}$, so that $M$ is a $\mathrm{II}_{1}$-factor. Assume that $M$ is not full. Since $M$ has property $\Gamma$, there is a sequence $\left(x_{n}\right)$ of unitaries in $M$ for which $\left\|z x_{n}-x_{n} z\right\|_{2} \rightarrow 0$ for all $z \in M$ and $\tau\left(e\left(x_{n}\right)\right)=0$ for all $n$. Since

$$
\left\|x_{n} v(g)-v(g) x_{n}\right\|_{2} \rightarrow 0 \text { for all } g \in G,
$$

the sequence $\left(x_{n}\right)$ satisfies, by (i), $\left\|x_{n}-e\left(x_{n}\right)\right\|_{2} \rightarrow 0$. The expectation $e$ satisfies $e\left(v(g) y v(g)^{*}\right)=v(g) e(y) v(g)^{*}$ for all $g \in G$ and $y \in M$. Therefore,

$$
\left\|v(g) e\left(x_{n}\right)-e\left(x_{n}\right) v(g)\right\|_{2} \rightarrow 0 \text { for all } g \in G .
$$

Since the action of $G$ is strongly ergodic on $N$, we have that

$$
\left\|e\left(x_{n}\right)\right\|_{2}=\left\|e\left(x_{n}\right)-\tau\left(e\left(x_{n}\right)\right) 1\right\|_{2} \rightarrow 0 .
$$

This contradicts the assumption that all $x_{n}$ are unitaries. Thus $M$ is a full $\mathrm{II}_{1}$-factor.

In the case that $N$ is a full $\mathrm{II}_{1}$-factor, the assumption of strong ergodicity for the action of $G$ is not necessary.

COROLLARY. Let $N$ be a full $\mathrm{II}_{1}$-factor and $G$ a countable group of automorphisms of $N$. If $G$ is not inner amenable, then the crossed product $M$ of $N$ by $G$ is a full $\mathrm{II}_{1}$-factor.

Proof. Let $\tau$ be the canonical trace on $N$. Since $N$ is a factor, by (i) in the Theorem, $M^{\prime} \cap M \subset \pi(N)^{\prime} \cap v(G)^{\prime} \cap M \subset \pi(N)^{\prime} \cap \pi(N)=\mathrm{Cl}$. Hence $M$ is a finite factor. Since $N$ is type $\mathrm{II}, M$ is a $\mathrm{II}_{1}$-factor. If $M$ is not full, there is a sequence $\left(x_{n}\right)$ of unitaries in $M$ for which $\tau\left(e\left(x_{n}\right)\right)=0$ for all $n$ and $\left\|x_{n} z-z x_{n}\right\|_{2} \rightarrow 0$ for all $z \in M$. By the same proof as (ii) in the Theorem, $\left\|x_{n}-e\left(x_{n}\right)\right\|_{2} \rightarrow 0$. On the other hand, the bounded sequence $e\left(x_{n}\right)$ satisfies $\left\|y e\left(x_{n}\right)-e\left(x_{n}\right) y\right\|_{2} \rightarrow 0$ for all $y \in$ $\pi(N)$, because $e$ is an expectation of $M$ onto $\pi(N)$. Therefore,

$$
\left\|e\left(x_{n}\right)\right\|_{2}=\left\|e\left(x_{n}\right)-\tau\left(e\left(x_{n}\right)\right) 1\right\|_{2} \rightarrow 0,
$$

because $N$ is full. This is a contradiction. Thus $M$ is a full $\mathrm{II}_{1}$-factor. 


\section{REFERENCES}

1. C. A. Akemann and M. Walter, Unbounded negative definite functions, Canad. J. Math. 33 (1981), 862-871.

2. M. Choda and Y. Watatani, Fixed point algebra and property T, Math. Japon. 27 (1982), 263-266.

3. M. Choda, Property T and fullness of the group measure space construction, Math. Japon. 27 (1982), 535-539.

4. A. Connes, Almost periodic states and factors of type $\mathrm{III}_{1}$, J. Funct. Anal. 16 (1974), 415-445.

5. A. Connes and B. Weiss, Property T and asymptotically invariant sequences, Israel J. Math. 37 (1980), 209-210.

6. E. G. Effros, Property $\Gamma$ and inner amenability, Proc. Amer. Math. Soc. 47 (1975), 483-486.

7. Y. Haga and $\mathrm{Z}$. Takeda, Correspondence between subgroups and subalgebras in a cross product von Neumann algebra, Tôhoku Math. J. 21 (1972), 167-190.

8. D. A. Kazhdan, Connection of the dual space of a group with the structure of its closed subgroups, Funct. Anal. Appl. 1 (1967), 63-65.

9. F. Murray and J. von Neumann, On rings of operators. IV, Ann. of Math. 44 (1943), 716-808.

Department of Mathematics, Osaka Kyoiku University, Tennoji, Osaka 543, Japan 